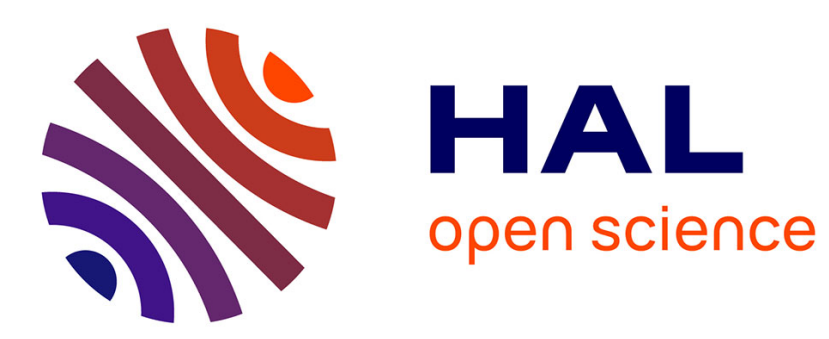

\title{
Réponse d'un photodétecteur à des signaux brefs
}

R. Papoular

\section{To cite this version:}

R. Papoular. Réponse d'un photodétecteur à des signaux brefs. Revue de Physique Appliquée, 1968, 3 (2), pp.169-174. 10.1051/rphysap:0196800302016900 . jpa-00242841

\section{HAL Id: jpa-00242841 https://hal.science/jpa-00242841}

Submitted on 1 Jan 1968

HAL is a multi-disciplinary open access archive for the deposit and dissemination of scientific research documents, whether they are published or not. The documents may come from teaching and research institutions in France or abroad, or from public or private research centers.
L'archive ouverte pluridisciplinaire HAL, est destinée au dépôt et à la diffusion de documents scientifiques de niveau recherche, publiés ou non, émanant des établissements d'enseignement et de recherche français ou étrangers, des laboratoires publics ou privés. 


\title{
RÉPONSE D’UN PHOTODÉTEGTEUR A DES SIGNAUX BREFS
}

\author{
Par R. PAPOULAR, \\ Groupe de Recherches de l'Association EURATOM-GEA sur la Fusion, 92-Fontenay-aux-Roses, France.
}

(Reçu le 22 mars 1968.)

\begin{abstract}
Résumé. - On considère une chaîne de photodétection composée d'un photomultiplicateur et d'un circuit $R C$. La réponse de cette chaîne est calculée pour divers types de signaux usuels. Il en découle une méthode de mesure des caractéristiques essentielles de la chaîne. L'influence de l'effet de grenaille sur les régimes transitoires et permanents est prise en considération. Nous proposons enfin quelques règles pour l'interprétation de la réponse à un signal inconnu.

Abstract. - The photodetector considered here comprises a photomultiplier and an $R C$ circuit. The time response of this device is computed for different, common shapes of light signals. This leads to a procedure for the determination of the essential parameters of the detector. The influence of shot effects on transient and permanent regimes is considered. We also discuss some of the rules for the interpretation of the detector response to an unknown signal.
\end{abstract}

1. Introduction. - L'avènement des lasers à impulsions géantes a suscité l'étude d'émissions lumineuses de courte durée ou à front de montée raide. Le problème est quelquefois compliqué par la faiblesse de l'éclairement à mesurer. Dans ces cas, l'interprétation des signaux détectés est assez délicate, et cela d'autant plus que les temps caractéristiques de l'émission lumineuse sont plus voisins de ceux de la chaîne de détection utilisée. Il y a là une analogie assez remarquable avec le problème posé en spectroscopie par la convolution d'un profil de raie fine avec une fonction d'appareil de largeur voisine.

Dans cet article, nous calculons la réponse d'un photodétecteur à certains types de signaux usuels et nous recherchons les moyens de remonter, d'un oscillogramme enregistré, à la forme de l'impulsion lumineuse qui l'a engendré.

Le type de photodétecteur considéré est un photomultiplicateur rapide, tel le 56 AVP de la Radiotechnique. La méthode de calcul et les résultats sont néanmoins applicables à d'autres cas.

2. Réponses transitoire et percussionnelle. - La chaîne de photodétection doit être considérée comme un tout et il est incorrect de calculer séparément, pour les multiplier ensuite l'une par l'autre, la réponse du PM (photomultiplicateur) et celle de l'amplificateur ou de l'oscilloscope qui le suit. Du point de vue de la réponse aux signaux brefs, ce dernier composant peut être assimilé à un circuit $R C$ passe-bas. Le type de chaîne de photodétection que nous considérons ici pourra donc être représenté par le schéma de la

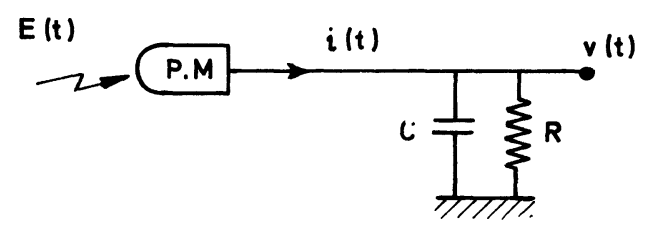

FIG. 1.

figure 1 , où $E$ est l'éclairement, $i$ le courant débité par le PM et $v$ la tension enregistrée.

La réponse globale de la chaîne à un signal quelconque peut être calculée à partir de la réponse transitoire $A_{\mathrm{G}}(t)$, définie comme étant la tension de sortie correspondant à un éclairement $E=U(t)$ (photons par seconde) où $U$ est l'échelon unité. On peut également partir de la réponse percussionnelle, $A_{\mathrm{G}, \delta}(t)$, qui correspond à un éclairement en forme de fonction de Dirac, $\delta(t)$.

Nous allons calculer $A_{\mathrm{G}}$ et $A_{\mathrm{G}, \delta}$.

2.1. RéPonse transitoire $A_{\mathrm{G}}(t)$. - Un éclairement $U(t)$ provoque, dans le circuit anodique du PM, le passage d'un courant $i=A_{\mathrm{p}}(t)$, égal, par définition, à la réponse transitoire du PM. On sait ([1], p. 451) que la tension fournie par la chaîne est alors :

$$
v(t)=A_{\mathrm{G}}(t)=\frac{\mathrm{d}}{\mathrm{d} t} \int_{0}^{t} A_{\beta}(\tau) A_{\mathrm{p}}(t-\tau) \mathrm{d} \tau
$$

où $A_{\beta}(t)$ est la réponse transitoire du circuit $R C$, c'est-à-dire :

$$
A_{\beta}(t)=R\left(1-\mathrm{e}^{-\beta t}\right)
$$


où $\beta=1 / R C$ et où il est sous-entendu que $A_{\beta}(t)=0$ pour $t<0$ (comme pour toutes les fonctions du temps dans ce qui suit).

D'autre part, on admet [2] que la réponse percussionnelle du photomultiplicateur est :

$$
A_{\mathrm{p}, \delta}(t)=\frac{I_{0}}{\sqrt{\pi}} \exp -\left(\frac{t-t_{0}}{\tau_{0}}\right)^{2}
$$

où $I_{0}=\frac{Q_{0}}{\tau_{0}}=\frac{r N_{0} q}{\tau_{0}}, r=$ rendement quantique de la photocathode, $N_{0}=$ coefficient de multiplication du photomultiplicateur, $q=$ charge de l'électron, $\tau_{0}=$ dispersion du temps de transit, $t_{0}=$ temps de transit moyen.

La réponse transitoire du photomultiplicateur est la primitive de $A_{\mathrm{p}, \delta}$, et sera donnée par :

$A_{\mathrm{p}}(t)=\int_{0}^{t} A_{\mathrm{p}, \delta} \mathrm{d}(t)=\frac{Q_{0}}{2}\left[\Theta\left(\frac{\tau_{0}}{t_{0}}\right)+\Theta\left(\frac{t-t_{0}}{\tau_{0}}\right)\right]$.

Le calcul de (1) donne alors :

$$
\begin{aligned}
A_{\mathrm{G}}(t)= & \frac{R Q_{0}}{2}\left\{\Theta\left(\frac{t_{0}}{\tau_{0}}\right)+\Theta\left(\frac{t-t_{0}}{\tau_{0}}\right)\right. \\
& \left.-\left[\Theta\left(\frac{T_{1}}{\tau_{0}}\right)+\Theta\left(\frac{t-T_{1}}{\tau_{0}}\right)\right] \mathrm{e}^{-\beta\left(t-T_{2}\right)}\right\}
\end{aligned}
$$

où $\Theta(\xi)$ est la fonction d'erreur, $\frac{2}{\sqrt{ } \pi} \int_{0}^{\xi} \mathrm{e}^{-\xi^{2}} \mathrm{~d} \xi$.

$$
T_{1}=t_{0}+\frac{\beta \tau_{0}^{2}}{2}, \quad T_{2}=t_{0}+\frac{\beta \tau_{0}^{2}}{4} .
$$

Si $\tau_{0} \rightarrow 0$ :

$$
\begin{aligned}
& A_{\mathrm{G}} \rightarrow \frac{R Q_{0}}{2}\left[\Theta\left(\frac{t_{0}}{\tau_{0}}\right)+\Theta\left(\frac{t-t_{0}}{\tau_{0}}\right)\right] {\left[1-\mathrm{e}^{-\beta\left(t-t_{0}\right)}\right] } \\
&=A_{\mathrm{p}}(t) \cdot A_{\beta}\left(t-t_{0}\right) .
\end{aligned}
$$

2.2. Réponse PergussionNelle $A_{\mathrm{G}, \delta}(t)$. - On sait que :

$$
A_{\mathrm{G}, \delta}(t)=\frac{\mathrm{d}}{\mathrm{d} t}\left[A_{\mathrm{G}}(t)\right] .
$$

Il en résulte que :

$$
A_{\mathrm{G}, \delta}(t)=\frac{Q_{0}}{2 C}\left[\Theta\left(\frac{T_{1}}{\tau_{0}}\right)+\Theta\left(\frac{t-T_{1}}{\tau_{0}}\right)\right] \mathrm{e}^{-\left(\frac{t-T_{2}}{R C}\right)} .
$$

Si l'on note que $T_{1}=t_{0}+\frac{\beta \tau_{0}^{2}}{2} \gg \tau_{0}$ en général, on voit que $\Theta\left(T_{1} / \tau_{0}\right) \simeq 1$ et que ce résultat est identique à celui de R. Parrot [3]. Il peut se mettre sous la forme, facile à exploiter :

$$
A_{\mathrm{G}, \delta}(\zeta)=\frac{Q_{0}}{2 C} \mathrm{e}^{-\frac{m^{2}}{4}} \cdot \mathrm{e}^{-m \zeta}[1+\Theta(\zeta)]
$$

où $m=\beta \tau_{0}=\tau_{0} / R C$ et $\zeta=\left(t-T_{1}\right) / \tau_{0}$.
Avec ces notations :

$$
\begin{aligned}
A_{\mathrm{G}}(\zeta)=\frac{R Q_{0}}{2}\{1+ & \Theta\left(\zeta+\frac{m}{2}\right) \\
& \left.-[1+\Theta(\zeta)] \mathrm{e}^{-m\left(\zeta+\frac{m}{4}\right)}\right\} .
\end{aligned}
$$

2.3. Formes limites De $A_{\mathrm{G}}$ ET $A_{\mathrm{G}, \delta}$ :

Si $R C \gg \tau_{0}(m \ll 1)$ :

$$
\begin{aligned}
& A_{\mathrm{G}} \rightarrow \frac{R Q_{0}}{2}\left[1+\Theta\left(\frac{t-t_{0}}{\tau_{0}}\right)\right]\left[1-\mathrm{e}^{-\left(\frac{t-t_{0}}{R C}\right)}\right] \\
& A_{\mathrm{G}, \delta} \rightarrow \frac{Q_{0}}{2 C}\left[1+\Theta\left(\frac{t-t_{0}}{\tau_{0}}\right)\right] \mathrm{e}^{-\left(\frac{t-t_{0}}{R C}\right)}
\end{aligned}
$$

Si $R C \ll \tau_{0}(m \gg 1)$ :

$$
\begin{aligned}
& A_{\mathrm{G}} \rightarrow \frac{R Q_{0}}{2}\left[1+\Theta\left(\frac{t-t_{0}}{\tau_{0}}\right)\right] \\
& A_{\mathrm{G}, \delta} \rightarrow R Q_{0} \cdot \frac{\mathrm{e}^{-\left(\frac{t-t_{0}}{\tau_{0}}\right)^{2}}}{\sqrt{\pi} \tau_{0}} .
\end{aligned}
$$

Nous avons calculé $A_{\mathrm{G}, \delta}$ pour quelques valeurs de $m$ comprises entre 1/4 et 2 . La figure 2 représente schématiquement l'évolution de $A_{\mathrm{G}, \delta}$ en fonction de $m$.

La réponse percussionnelle, $A_{\mathrm{G}, \delta}$, tire son importance du fait que, comme on le verra au para-
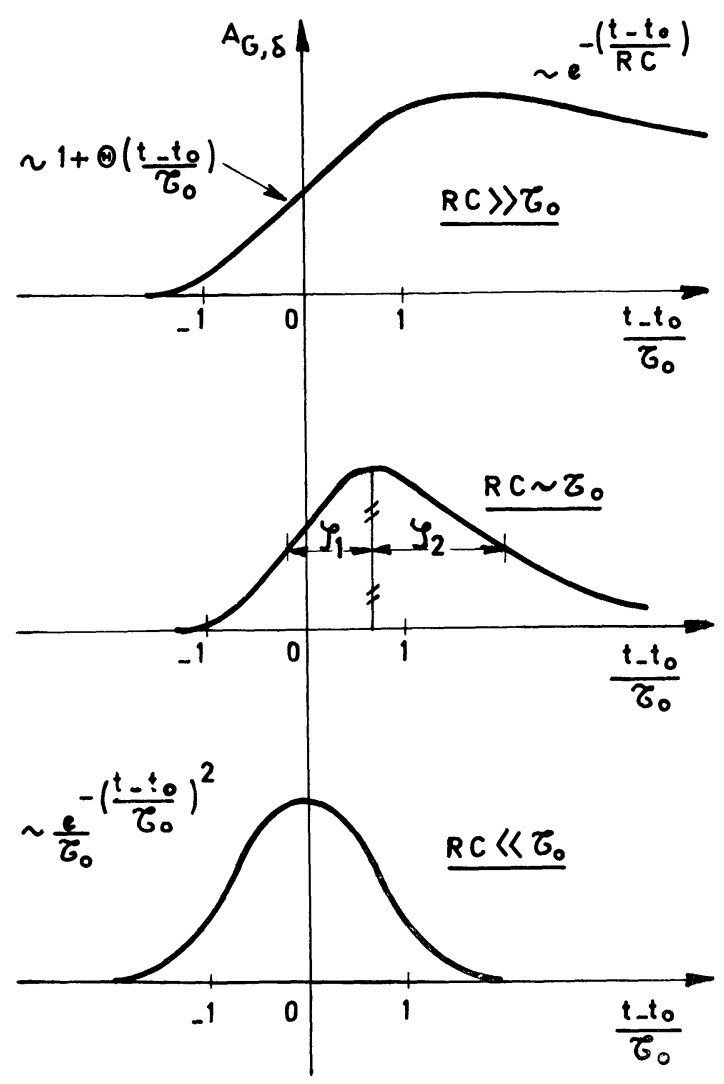

FIG. 2. 
graphe 3, il est facile d'en déduire les caractéristiques essentielles de la chaîne de détection. En outre, sa détermination expérimentale est particulièrement aisée : les thermoélectrons, ou électrons de bruit que la photocathode émet spontanément d'une manière aléatoire, constituent autant d'impulsions de Dirac, donnant naissance à une tension $A_{\mathrm{G}, \delta}(t)$ en sortie de la chaîne.

Notons que les réponses transitoires et impulsionnelles du photomultiplicateur et du circuit $R C$, considérés séparément, s'obtiennent à partir de (11), (12) en y posant $R C=0$, et de $(9),(10)$ en y posant $\tau_{0}=0$.

3. Détermination expérimentale de $\tau_{0}$ et $R G$. Le relevé de $A_{\mathrm{G}, \delta}(t)$ permet de déterminer $\tau_{0}$ et $R C$. En effet, l'exploitation numérique de (7) nous a amenés à tracer les courbes de la figure 3 , lesquelles représentent les variations, en fonction de $m=\tau_{0} / R C$, de $\zeta_{1}, \zeta_{2}$ et $\Delta \zeta=\zeta_{1}+\zeta_{2}, \zeta_{1}$ et $\zeta_{2}$ étant définis par la figure 2. De la figure 3 , connaissant $\zeta_{2} / \zeta_{1}$, on tire $m$.

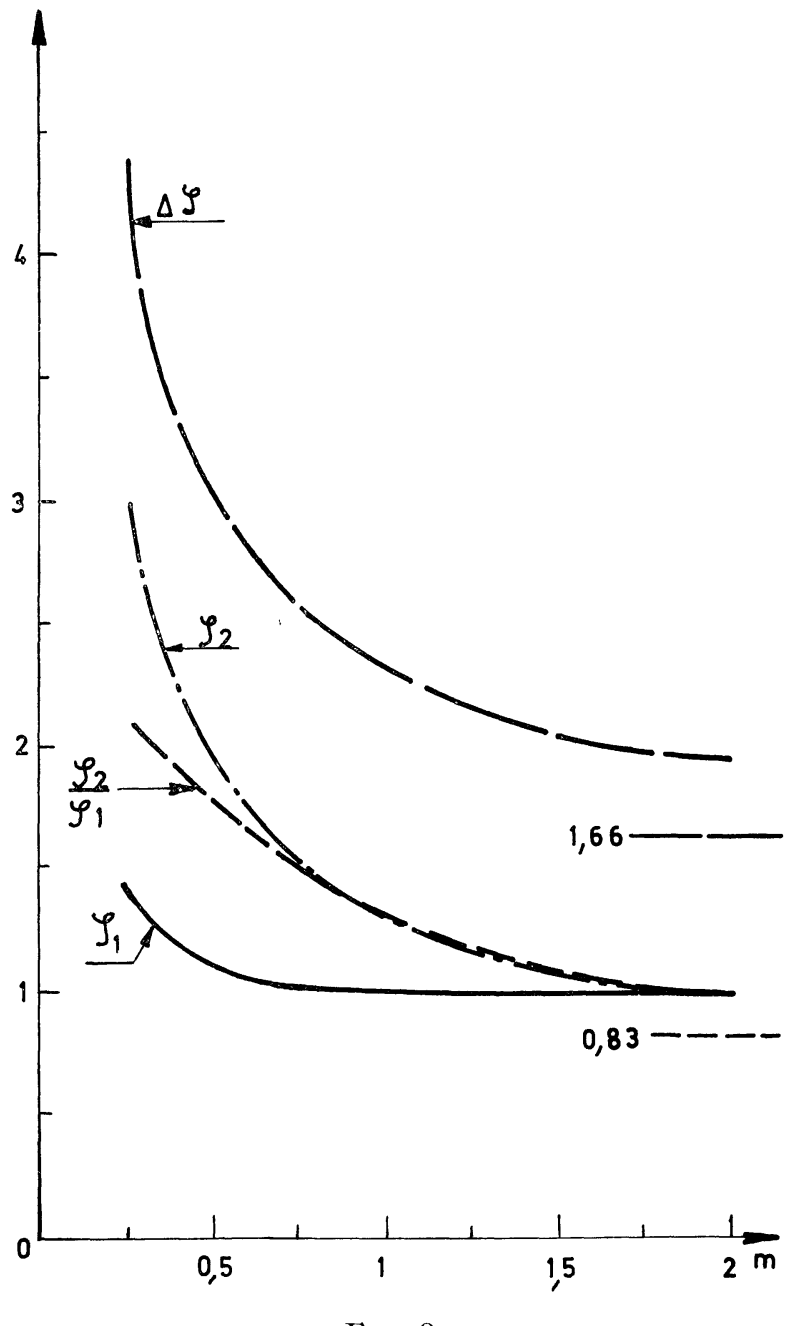

FIG. 3
S'aidant alors de la courbe, on déduit $\Delta \zeta$. La comparaison de $\Delta \zeta$ avec la largeur à mi-hauteur, $\Delta \tau$, lue sur le relevé de $A_{\mathrm{G}, \delta}$, donne $\tau_{0}=\Delta \tau / \Delta \zeta$; finalement, on peut calculer $R C=\tau_{0} / \mathrm{m}$.

On voit que, en principe, la connaissance de $R C$ n'est pas nécessaire pour déterminer $\tau_{0}$. Malheureusement, la précision de la détermination de $\tau_{0}$ par cette méthode décroît rapidement quand $m \rightarrow 0$, de même que celle de $R C$, quand $m \rightarrow \infty$.

4. Réponse globale à un sígnal quelconque. La connaissance de $\tau_{0}$ et $R C$ permet, en principe, de calculer la réponse globale $v(t)$ à un éclairement quelconque, $E(t)$ photons par seconde, à partir de la réponse transitoire (5) ou percussionnelle (6), et de l'une des relations suivantes ([1], p. 456) :

$$
\begin{gathered}
v(t)=\frac{\mathrm{d}}{\mathrm{d} t} \int_{0}^{t} A_{\mathrm{G}}(\tau) E(t-\tau) \mathrm{d} \tau \\
v(t)=\frac{\mathrm{d}}{\mathrm{d} t} \int_{0}^{t} A_{\mathrm{G}}(t-\tau) E(\tau) \mathrm{d} \tau \\
v(t)=A_{\mathrm{G}}(t) E(0)+\int_{0}^{t} A_{\mathrm{G}}(t-\tau) \frac{\mathrm{d} E(\tau)}{\mathrm{d} \tau} \mathrm{d} \tau \\
v(t)=A_{\mathrm{G}}(t) E(0)+\int_{0}^{t} A_{\mathrm{G}}(\tau) \frac{\mathrm{d} E(t-\tau)}{\mathrm{d}(t-\tau)} \mathrm{d} \tau \\
v(t)=A_{\mathrm{G}}(0) E(t)+\int_{0}^{t} A_{\mathrm{G}, \delta}(t-\tau) E(\tau) \mathrm{d} \tau \\
v(t)=A_{\mathrm{G}}(0) E(t)+\int_{0}^{t} A_{\mathrm{G}, \delta}(\tau) E(t-\tau) \mathrm{d} \tau
\end{gathered}
$$

qui sont équivalentes. Comme $A_{\mathrm{G}}(0)=0$, les transformées de Laplace de (17) et (18) s'écrivent, d'après le théorème de Borel ([1], p. 467) :

$$
\mathscr{V}(p)=\mathscr{A}_{\mathrm{G}, \delta}(p) \cdot \mathscr{E}(p) .
$$

On obtiendrait de même, en transformant (13) et $(14)$ :

$$
\mathscr{V}(p)=p \mathscr{A}_{\mathrm{G}}(p) \cdot \mathscr{E}(p)
$$

forme équivalente à (19) puisque $A_{\mathrm{G}, \delta}(t)=\frac{\mathrm{d}}{\mathrm{d} t} A_{\mathrm{G}}(t)$. Ce passage aux images symboliques simplifie quelquefois les calculs. Il permet aussi d'énoncer des propositions générales intéressantes.

En effet ([1], p. 464) :

$$
\operatorname{Lim}_{t \rightarrow \infty} v(t) \sqsupset \operatorname{Lim}_{k \rightarrow 0} k \mathscr{V}(k p)
$$

où le signe $\sqsupset$ a le sens de « original de ». Or, dans le cas présent, la transformation symbolique de (1) donne :

$$
\mathscr{A}_{\mathrm{G}}(p)=p \mathscr{A}_{\beta}(p) \mathscr{A}_{\mathrm{p}}(p)=\mathscr{A}_{\beta}(p) \cdot \mathscr{A}_{\mathrm{p}, \delta}(p)
$$

où, d'après (2) et (3) :

$$
\mathscr{A}_{\beta}(p)=\frac{1}{C} \cdot \frac{1}{p} \cdot \frac{1}{p+\beta}
$$

et :

$$
\mathscr{A}_{\mathrm{p}, \delta}(p)=\frac{Q_{0}}{2} \mathrm{e}^{\left(\frac{\tau_{0} p}{2}\right)^{2}}\left[1-\Theta\left(\frac{\tau_{0} p}{2}\right)\right] \mathrm{e}^{-t_{0} p}
$$


Il en résulte que :

$$
\begin{aligned}
& k \mathscr{V}(k p) \\
& =\frac{Q_{0}}{2 C} \cdot k \cdot \frac{1}{k p+\beta} \cdot \mathrm{e}^{\left(\frac{\tau_{0} k p}{2}\right)^{2}}\left[1-\Theta\left(\frac{k \tau_{0} p}{2}\right)\right] \mathrm{e}^{-t_{0} k p} \mathscr{E}(k p) .
\end{aligned}
$$

D'où :

$$
\operatorname{Lim}_{t \rightarrow \infty} v(t) \sqsupset \operatorname{Lim}_{k \rightarrow 0} \frac{R Q_{0}}{2}\left[k \frac{\mathrm{e}^{t_{0} k p} \mathscr{E}(k p)}{k p+\beta}\right] .
$$

La formule (22) montre que, après l'extinction $d u$ transitoire, la réponse $v(t)$ a la même forme que l'excitation $E(t)$. C'est là un résultat commun à toutes les chaînes passe-bas.

En ce qui concerne la durée du transitoire, (5) montre qu'il commence à $t=0$ et qu'il s'est éteint (à $15 \%$ près) lorsque $t \approx t_{0}+\tau_{0}$ ou $t \widetilde{>} t_{0}+2 R C$, selon que $\tau_{0}>R C$ ou $\tau_{0}<R C$.

5. Effet de grenaille. - Les considérations précédentes ne tiennent pas compte de la nature corpusculaire $\mathrm{du}$ rayonnement incident et $\mathrm{du}$ caractère aléatoire du flux de photons. Il faut pourtant les prendre en considération lorsque l'éclairement est relativement faible, en particulier au début de tout signal lumineux.

Tant que $E(t) \ll \frac{1}{2\left(\tau_{0}+\frac{1}{\beta}\right)}$, c'est-à-dire tant que deux impulsions ne peuvent se chevaucher, le signal $v(t)$ est composé de pics isolés de la forme $A_{\mathrm{G}, \delta}(t)$, avec une dispersion d'amplitude due au caractère aléatoire de la multiplication électronique.

Quand $E(t) \sim \frac{1}{2\left(\tau_{0}+\frac{1}{\beta}\right)}$, le signal $v(t)$ est d'autant plus complexe qu'au chevauchement des impulsions vient s'ajouter la dispersion des intervalles entre impulsions successives. Une analyse graphique de l'empilement permet néanmoins d'affirmer que le pied de la courbe représentative de $v(t)$ aura toujours la forme $d u$ front de montée de $A_{\mathrm{G}, \delta}(t)$. Cette portion de courbe, dont la durée ne peut excéder $\sim 2 \tau_{0}$, sera suivie d'une période

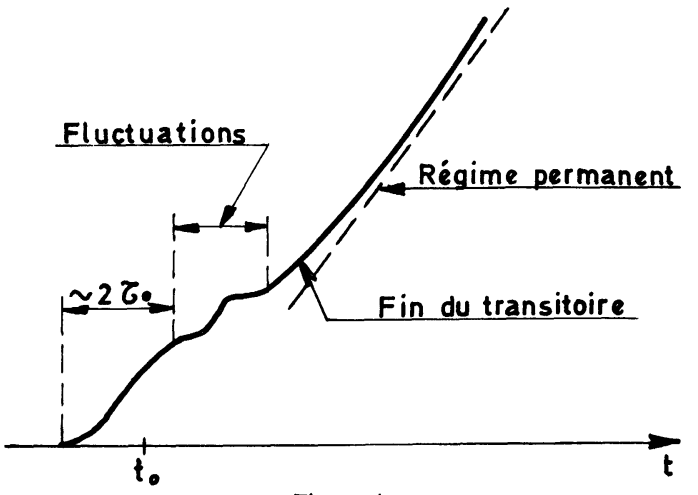

FIG. 4. de fluctuations, d'autant plus brève que $\mathrm{d} E / \mathrm{d} t$ est plus grand ( fig. 4).

Enfin, lorsque $E(t) \gg \frac{1}{2\left(\tau_{0}+\frac{1}{\beta}\right)}$, il est possible d'utiliser les résultats des paragraphes précédents. Il faut remarquer, toutefois, que ces résultats ne fournissent qu'une valeur moyenne du signal attendu. En réalité, toujours par suite de l'effet de grenaille, $v(t)$ est sujet à des fluctuations désordonnées, d'écart quadratique moyen :

$$
\sqrt{\overline{\overline{v_{\mathrm{b}}^{2}}}}=R \sqrt{\overline{\overline{i_{\mathrm{b}}^{2}}}}=R \sqrt{2 Q_{0} \bar{I} B(1+M)}
$$

où $Q_{0}=r N_{0} q$ (voir équ. (3)), $\bar{I}=\frac{\mathscr{V}}{R}, \quad B=\frac{1}{R C}$ $=$ bande passante de la chaîne de détection, et $M=\left(\mathrm{e}^{h \mathrm{v} / k T}-1\right)^{-1}$, où $\nu$ et $T$ sont la fréquence et la température du rayonnement incident [4]. Un exemple numérique montrera que ces fluctuations peuvent être importantes, même si le rayonnement incident est intense $\left({ }^{\mathbf{1}}\right)$. Considérons un rayonnement tel que $h \nu=2,5 \mathrm{eV}$ et $k T=10 \mathrm{eV}$, donc $M=4$. Si le photomultiplicateur est un 56 AVP de rendement quantique $r=20 \%$, fonctionnant avec un gain de $10^{8}$ et débitant sur un circuit composé de $R=100 \Omega$ et $C=100 \mathrm{pF}$, la relation (23) montre que :

$$
\frac{\sqrt{\overline{v_{\mathrm{b}}^{2}}}}{v}=\frac{\sqrt{\overline{i_{\mathrm{b}}^{2}}}}{\bar{I}}=\frac{\sqrt{2 Q_{\mathrm{o}} B(1+M)}}{\bar{I}}=\sqrt{\frac{3}{10^{3} \bar{I}}} .
$$

Mais $I$ ne peut excéder $100 \mathrm{~mA}$ sans saturer le photomultiplicateur. Il en résulte que, au mieux, $\sqrt{\overline{v_{\mathrm{b}}^{2}}} / v \sim 17 \%$.

6. Réponse à quelques signaux usuels. - L'étude de la réponse de la chaîne à quelques signaux usuels facilitera l'interprétation des résultats expérimentaux.

6.1. RÉPONSE A UNE « RAMPE », $E_{1}(t)=a_{1} t . U(t)$. - La combinaison de (5) avec (13) fournit :

$$
\begin{aligned}
& v_{1}(t)=\frac{R Q_{0} a_{1}}{2}\left\{t+\int_{0}^{t} \Theta\left(\frac{t-t_{0}}{\tau_{0}}\right) \mathrm{d} \tau\right. \\
& +R C\left[1+\Theta\left(\frac{t-t_{0}-\tau_{0}^{2} / 2 R C}{\tau_{0}}\right)\right] \\
& \left.\exp \left[-\frac{1}{R C}\left(t-t_{0}-\frac{\tau_{0}^{2}}{4 R C}\right)\right]-R C\left[1+\Theta\left(\frac{t-t_{0}}{\tau_{0}}\right)\right]\right\} .
\end{aligned}
$$

D’après (22) :

$\operatorname{Lim}_{t \rightarrow \infty} v_{1}(t)=R Q_{0} a_{1}\left(t-t_{0}-R C\right) \sim R Q_{0} a_{1}\left(t-t_{0}\right)$.

\subsection{RÉponse A UNE EXPONENTIELle :}

$$
E_{2}(t)=a_{2} \mathrm{e}^{\gamma t} U(t) \text {. }
$$

(1) C'est M. Nussli (L.E.P., Limeil) qui a attiré mon attention sur ce fait. 
En procédant de la même manière, on trouve :

$$
\begin{aligned}
v_{2}(t)= & \frac{R Q_{0} a_{2}}{2(\gamma R C+1)}\left\{\mathrm{e}^{\gamma\left(t-t_{0}+\gamma \frac{\tau_{0}^{2}}{4}\right)}\right. \\
& {\left[1+\Theta\left(\frac{\left.t-t_{0}+\gamma \frac{\tau_{0}^{2}}{2}\right)}{\tau_{0}}\right)\right]-\mathrm{e}^{-\frac{1}{R C}\left(t-t_{0}-\frac{\tau_{0}^{2}}{4 R C}\right)} } \\
& {\left.\left[1+\Theta\left(\frac{t-t_{0}-\frac{\tau_{0}^{2}}{2 R C}}{\tau_{0}}\right)\right]\right\} }
\end{aligned}
$$

$$
v_{2}(t) \simeq \frac{R Q_{0} a_{2}}{2(\gamma R C+1)}
$$

$$
\begin{aligned}
& {\left[\mathrm{e}^{+\gamma\left(t-t_{0}\right)}-\mathrm{e}^{-\left(\frac{t-t_{0}}{R C}\right)}\right]\left[1+\Theta\left(\frac{t-t_{0}}{\tau_{0}}\right)\right]} \\
& \operatorname{Lim}_{t \rightarrow \infty} \mathscr{V}_{2}(t)=\frac{R Q_{0} a_{2}}{\gamma R C+1} \mathrm{e}^{\gamma\left(t-t_{0}\right)} .
\end{aligned}
$$

On vérifie, sur ces deux cas, que, au transitoire près, $v(t)$ a la même forme que $E\left(t-t_{0}\right)$.

\subsection{RÉPONSE A UNE GAUSSIENNE :}

$$
E_{3}(t)=\frac{a_{3}}{T \sqrt{\pi}} \mathrm{e}^{-\left(\frac{t-t_{\mathrm{i}}}{T}\right)^{2}} .
$$

La combinaison de (6) avec (17) donne :

$$
\begin{aligned}
v_{3}(t)=\frac{Q_{0} \mathrm{e}^{-\frac{m^{2}}{4}} a_{3} \tau_{0}}{2} \mathrm{e}^{-m \zeta} & 2 \sqrt{\pi} C T \\
& \int_{0}^{\zeta} \mathrm{e}^{-\left(\frac{Z-Z_{\mathrm{i}}}{S}\right)^{2}+m Z}[1+\Theta(\zeta-Z)] \mathrm{d} Z
\end{aligned}
$$

où $\quad m=\tau_{0} / R C, \quad \zeta=\frac{1}{\tau_{0}}\left(t-t_{0}-\frac{\beta \tau_{0}^{2}}{2}\right), \quad Z=\tau / \tau_{0}$, $Z_{\mathrm{i}}=t_{\mathrm{i}} / \tau_{0}, S=T / \tau_{0}$.

Si $T \ll \tau_{0}, \quad E_{3}(t)$ est une impulsion de Dirac et $\mathscr{V}_{3}(t) \simeq a_{3} A_{\mathrm{G}, \delta}\left(t-t_{\mathrm{i}}\right)$ est bien proportionnel à la réponse percussionnelle de notre chaîne.

Si $T \gg \tau_{0}$, on peut considérer que $\Theta(x)=-1$ pour $x<0$ et +1 pour $x>0$. Dans ces conditions :

$v_{3}(t) \simeq \frac{Q_{0} a_{3}}{2 C} \mathrm{e}^{-\frac{m^{2}}{4}} \cdot \mathrm{e}^{-\frac{m^{\prime 2}}{4}} \cdot \mathrm{e}^{-m^{\prime} \zeta^{\prime}}\left[1+\Theta\left(\zeta^{\prime}\right)\right]$

où $m^{\prime}=m S=T / R C$ et :

$$
\zeta^{\prime}=\frac{t-t_{0}-t_{\mathrm{i}}}{T}-\frac{T}{2 R C}\left(1+\frac{\tau_{0}^{2}}{T^{2}}\right) .
$$

Cette relation est exactement de la même forme que $A_{\mathrm{G}, \delta}$ (cf. équ. (7)). C'est dire qu'un signal incident gaussien n'est pas restitué sous une forme gaussienne, à moins que $T \gg R C$ (cf. $\S 2.3$ ).

On peut estimer l'erreur commise en utilisant (27). Posons pour cela :

$$
\begin{gathered}
\Theta(x)=x \text { pour }-1<x<1 \\
\Theta(x)=-1 \text { pour } x<1
\end{gathered}
$$$$
\text { et } \quad \Theta(x)=+1 \text { pour } x>1 \text {. }
$$

L'intégrale figurant dans (27) se calcule alors aisément et l'on trouve que la différence entre la valeur vraie de $v_{3}(t)$ et celle donnée par (27) est :

$$
\begin{array}{r}
\varepsilon(t)=-\frac{Q_{0} a_{3}}{2 C} \mathrm{e}^{-\left(\frac{m^{2}+m^{\prime 2}}{4}\right)} \cdot \mathrm{e}^{-m^{\prime} \zeta^{\prime}} \frac{1}{2} \\
\left\{\left(1-S \zeta^{\prime}\right)\left[\Theta\left(\zeta^{\prime}\right)-\Theta\left(\zeta-\frac{1}{S}\right)\right]\right. \\
\left.-\frac{S}{\sqrt{\pi}}\left[\mathrm{e}^{-\zeta^{\prime 2}}-\mathrm{e}^{-\left(\zeta^{\prime}-\frac{1}{S}\right)^{2}}\right]\right\} .
\end{array}
$$

On vérifie que $\varepsilon \rightarrow 0$ lorsque $S \rightarrow 0$ ou $S \rightarrow \infty$, c'est-à-dire lorsque $T \ll \tau_{0}$ ou $T \gg \tau_{0}$; dans les deux cas, l'expression (27) tend vers la valeur vraie de $v_{3}(t)$.

L'erreur est évidemment maximale pour $T \sim \tau_{0}$. Prenons par exemple le cas où $T \simeq R C\left(m \simeq m^{\prime} \simeq 1\right)$ et considérons l'instant $\zeta^{\prime}=0$, voisin du sommet de la courbe de réponse. Il vient :

$$
\left(\frac{\varepsilon}{v_{3}}\right)_{\zeta^{\prime}=0} \simeq 0,25 \text {. }
$$

Les choses se simplifient si $m \gg 1$, c'est-à-dire $\tau_{0} \gg R C$, car alors $A_{\mathrm{G}, \delta}(t)$ est une gaussienne $(\S 2.3)$ et $v_{3}(t)$ aussi, puisque cette dernière expression est le produit de composition d'une gaussienne par une autre (équ. (17)); de plus, la largeur à mi-hauteur $\Delta T$ de $v_{3}(t)$ sera donnée par :

$$
(\Delta T)^{2}=T^{2}+(\Delta \tau)^{2}
$$

où $\Delta \tau$ est la largeur à mi-hauteur de $A_{\mathrm{G}, \delta}(t)$, soit $\sim 1,66 \tau_{0}$ (cf. fig. 3).

7. Interprétation de la réponse d'une chaîne de détection. - Le problème le plus intéressant est celui qui consiste à remonter d'un oscillogramme au signal lumineux qui lui a donné naissance. Ce problème est théoriquement résolu par l'application de (19) ou (20) lorsque l'on connaît $A_{\mathrm{G}, \delta}(t)$ ou $A_{\mathrm{G}}(t)$. Il suffit en effet de déterminer, par une quadrature ([1], p. 483), l'originale de $\mathscr{V}(p) / \mathscr{A}_{\mathrm{G}, \delta}(p)$ ou celle de $\mathscr{V}(p) / p \mathscr{A}_{\mathrm{G}}(p)$. Mais les expressions de $\mathscr{V}, \mathscr{A}_{\mathrm{G}, \delta}$ et $\mathscr{A}_{\mathrm{G}}$ sont en général si compliquées que cette quadrature paraît impossible, sans parler des complications dues à l'effet de grenaille $(\S 5)$.

Si l'on dispose d'une machine à calculer, il est plus commode de procéder de la manière suivante [5] : Soient $\mathscr{A}_{\mathrm{G}, \delta}(\omega), \mathscr{E}(\omega)$ et $\mathscr{V}(\omega)$ les transformées de Fourier (ou spectres) de $A_{\mathrm{G}, \delta}(t), E(t)$ et $v(t)$. La transformée de Fourier de (17) ou (18) donne :

$$
\mathscr{E}(\omega)=\mathscr{V}(\omega) / \mathscr{A}_{\mathrm{G}, \delta}(\omega) \text {. }
$$

Il suffit alors de transformer $\mathscr{E}(\omega)$ pour obtenir $E(t)$. Ces transformations sont plus faciles à exécuter si l'on complète $A_{\mathrm{G}, \delta}, E$ et $v$ par leur symétrique par rapport à l'axe $t=0$ (cela est possible puisque ces fonctions sont nulles pour $t<0)$. Les spectres $\mathscr{A}_{\mathrm{G}, \delta}$, $\mathscr{E}$ et $\mathscr{V}$ ne contiennent plus, alors, que des termes en cosinus, ce qui réduit le calcul.

Dans certains cas particuliers, des simplifications 
sont possibles. Ainsi lorsque l'oscillogramme tend vers une forme asymptotique quand $t \rightarrow \infty$, l'application de (21) permet de calculer $\lim _{t \rightarrow \infty} E(t)$ assez
facilement.

Le problème est encore simplifié si $R C \gg \tau_{0}$ ou
$R C \ll \tau_{0}$ et naturellement davantage si ces deux temps $R C \ll \tau_{0}$ et naturellement davantage sices deux temps
sont très inférieurs aux temps caractéristiques de l'oscillogramme car, alors, $E(t) \simeq v(t)$.

Si l'inversion proprement dite de l'oscillogramme s'avère trop difficile, on peut encore comparer ce dernier, après lissage, avec les réponses à des fonctions courantes, telles que $A_{\mathrm{G}, \delta}, A_{\mathrm{G}}, v_{1}, v_{2}, v_{3}$ ou à d'autres fonctions, faciles à calculer [3]. Compte tenu de l'étroite analogie entre fonction d'appareil et réponse percussionnelle, on fera également son profit des résultats obtenus, en spectroscopie, dans l'étude de la convolution de diverses fonctions d'appareil avec différents profils de raies spectrales.

\section{BIBLIOGRAPHIE}

[1] Angot (A.), Compléments de Mathématiques, Revue d'Optique, 1949.

[2] LEWIS et WELLS, Millimicrosecond Pulse Techniques, Pergamon Press, 1959.

[3] Parrot (R.), C. R. Acad. Sc. Paris, 1966, 263 B, 795, équ. (1).
[4] Tolman (R. C.), The Principles of Statistical Mechanics, Oxford, 1938, p. 630.

[5] ARsaC (J.), Transformation de Fourier et théorie des distributions, Dunod, 1961, p. 209. 